\title{
Short and long term treatment of asthma with intravenous nutrients Welman A Shrader Jr*
}

\author{
Address: Santa Fe Center for Allergy \& Environmental Medicine, Director Santa Fe, USA \\ Email: Welman A Shrader* - wshradermd@aol.com \\ * Corresponding author
}

This article is available from: http://www.nutritionj.com/content/3/I/6

(C) 2004 Shrader; licensee BioMed Central Ltd. This is an Open Access article: verbatim copying and redistribution of this article are permitted in all media for any purpose, provided this notice is preserved along with the article's original URL.

\begin{abstract}
Background: Asthma is an increasing problem in this country and others. Although medications for the treatment of asthma abound and are improving, there are inherent risks and side effects with all of them. Intravenous magnesium has been employed in the treatment of acute asthma, but its use has not become universal, nor has it been studied for the treatment of chronic asthma. It is known to be a safe drug with minimal side effects. In this study, the author investigates the use of magnesium and other nutrients in the treatment of both acute and chronic asthma.
\end{abstract}

Methods: In this non-blinded outcome study, following informed consent, forty-three (43) randomly selected volunteer patients with both acute and chronic asthma were treated with IV infusions described herein. All patients were observed with spirometry 10 minutes post-infusion; two sub-groups of patients were also observed after multiple infusions over a short period of time (less than one month) and a longer period of time (average 5.8 months). Pulmonary function was analyzed by spirometric testing with pre- and post-infusion spirometric measurements with the pre/post group. For longer term (Trend) patients, baseline spirometry measurements were compared to spirometry measurements after patients had received multiple infusions over a period of time. Eight (8) patients were measured for both pre/post and Trend data.

Results: The 38 pre-infusion/post-infusion patients with acute and chronic asthma demonstrated an overall average improvement (percentage improvement in percent predicted) of $45 \%$. The 13 patients measured for improvement over time (Trend data, average duration 5.82 months), demonstrated an overall average improvement (percentage improvement in percent predicted) of $57 \%$. Of the 13 patients in the multiple infusion group, 9 patients who received longer-term therapy (average duration of 12.58 months) for chronic asthma demonstrated an overall average improvement of $95 \%$ (percentage improvement in percent predicted).

Conclusion: The use of intravenous treatment with multiple nutrients, including magnesium, for acute and chronic asthma may be of considerable benefit. Pulmonary function improved progressively the longer patients received treatment. 


\section{Background}

Asthma has been a major focus for physicians in recent years because both the incidence and the mortality appear to be increasing, especially within certain ethnic or geopolitical groups [1-3]. This increase has been blamed variously on different types of inhalers used for asthma, [4] chemical environmental pollution, mostly of the air, [5] increased reporting incidences and many other factors. No matter what the cause, the incidence of asthma has appeared to increase and to worsen over the years, even if it may appear to be more stable recently $[6,7]$.

In addition, asthma has become increasingly more difficult to treat. Several studies indicate the mortality of asthma is higher and that the incidence of status asthmaticus patients seen in emergency rooms has increased [8]. Despite many newer drugs for asthma, people are dying more frequently from this illness.

In view of these rather grim realizations and statistics, the author has undertaken research of a modality in an attempt to mitigate the effects of asthma. A most promising treatment appears to be intravenous therapy with magnesium and other nutrients, both for the acute and chronic illness.

The use of IV parenteral nutrient therapy for asthma was begun in the author's office in the late 1980's, soon after the first papers appeared in the literature involving the use of IV magnesium for the treatment of acute asthma [9]. Many papers have followed since then, but they have concentrated primarily on intravenous magnesium, usually in the sulfated form [10-35]. Most of these authors have found that magnesium sulfate, via IV infusion, is beneficial for the treatment of asthma, and often extremely so. However, not all study results have been positive. This may have been due to the way the infusions were administered, the dose employed, or other factors such as severity of disease.

The author's IV protocols were expanded based on studies of the theoretical and known effects of various other nutrients on both acute and chronic asthma, as a result of previous work with general parenteral nutrients. Dr. Jonathan Wright's work with molybdenum [36] for the treatment of chronic asthma, as well as his and others' work with vitamin B-12, $[37,38]$ further influenced this study. Based on the early studies with magnesium and the work with molybdenum and B-12, the author undertook to employ other added nutrients to test the combined clinical efficacy.

\section{Methods}

This study began with a total of 49 patients who came to the author's clinic for treatment from 1989 through 1998.
These patients all had moderate to severe (steroiddependent) asthma, and all required one or more medications for asthma. Patients were asked to volunteer for the study, and all signed appropriate informed consent documents. Not all patients treated in the clinic volunteered for the study, and no patient who volunteered was excluded unless therapy appeared to fail.

During the study, 6 patients showed no initial response to the first treatment. These 6 patients were considered nonresponders and were not included in the data pool in this study, since there was no measurable improvement with a trial infusion. Forty-three (43) patients of mixed Caucasian descent were eventually enrolled, consisting of 16 males and 27 females, with a combined average age of 53 years.

Patients evaluated for pre- and post infusion results were given the IV "Push" protocol indicated in Table 1. These patients were evaluated at random times at the clinic; some were in mild to moderate distress. This IV was administered by a nurse with a $35 \mathrm{cc}$ BD syringe and a 2325 -gauge butterfly needle attached, with the amount of sterile water indicated. The IV Push infusions were administered over 10-15 minutes, rapidly enough to cause the patient to experience some sensation of warmth and flushing, but not rapidly enough to cause significant hypotension. Patients in the longer-term group ("Trend") were given one Push protocol initially. Subsequent to this, the "Infusion" protocol was given as an IV infusion in 250 cc of Sterile Water, generally over 45-60 minutes. Sterile Water was used to keep osmolarity in tolerable ranges. Of the thirteen (13) patients in trend group, eight were also evaluated in the pre/post group, while five (5) were evaluated only for Trend data. In the Trend group, the pre/ post data was taken from the initial IV session.

Thirteen (13) patients were given the Infusion (Table 1) protocol and evaluated for variable lengths of time with serial spirometry testing for Trend data. Spirometry trend data was obtained using a baseline pulmonary function test and comparing data obtained from that test with the results of the spirometry results at the end of the treatment period.

All patients were evaluated pre- and post-IV treatment with spirometry (pulmonary function testing, or PFT), using the SX-Platinum ${ }^{\circledast}$ computerized pulmonary function testing program. Spirometry was done immediately (within 10 minutes) after the protocols were administered. The computerized results were based on predicted Knudson normal for age, sex, weight and height.

The Trend group was further divided into "Short Term" and "Long Term" groups. The Short Term group consisted 
Table I: Intravenous Protocols Used for Acute and Chronic Asthma

\begin{tabular}{|c|c|c|c|}
\hline Nutrient & $\mathrm{mOsm} / \mathrm{ml}$ & "Push" Protocol (ml) & Infusion Protocol (ml) \\
\hline Ascorbic acid $500 \mathrm{mg} . / \mathrm{ml}$ & 5.8 & 3 & 12 \\
\hline B-12 (hydroxycobalamine) $1000 \mathrm{mcg} . / \mathrm{ml}$ & .30 & I & 1 \\
\hline B-6 (pyridoxine) $100 \mathrm{mg} . / \mathrm{ml}$ & 1.11 & 2 & 5 \\
\hline B-complex $100 \mathrm{mg} . / \mathrm{ml}$ & 2.14 & 2 & 2 \\
\hline Calcium gluconate $10 \%(100 \mathrm{mg} . / \mathrm{ml})$ & .72 & 2 & 2 \\
\hline Magnesium sulfate $500 \mathrm{mg} . / \mathrm{ml}$ & 4.06 & $4-5$ & $5-6$ \\
\hline Mineral mix ** & .57 & & I \\
\hline Molybdenum 500 mcg./ml & .10 & & 1 \\
\hline Pantothenic acid ("B-5") 250 mg./ml & .85 & 2 & 2 \\
\hline Sterile Water & 0 & $13-14$ & 250 \\
\hline
\end{tabular}

** Mineral mix formulation: Copper I mg./ml, chromium $50 \mathrm{mcg} . / \mathrm{ml}$, manganese $100 \mathrm{mcg} . / \mathrm{ml}$, molybdenum $250 \mathrm{mg} . / \mathrm{ml}$, selenium $200 \mathrm{mcg} . / \mathrm{ml}$, vanadium $100 \mathrm{mcg} . / \mathrm{ml}$, zinc $5 \mathrm{mg} . / \mathrm{ml}$.

of those patients who had "trend" data measured for one month or less, while those in the Long Term group were treated for up to 19 months. There were 4 patients in the Short Term group, 3 females and 1 male, average age 62, with an average duration of therapy of 0.43 mos. and 3.25 infusions per patient. There were 9 patients in the Long Term group, 6 females and 3 males, average age 53, with an average duration of therapy 12.58 mos. and 9.25 infusions per patient.

The average length of treatment periods for combined Trend patients was 5.82 months, with the shortest being 0.2 months. IV's were given anywhere from twice weekly to once every few months, with an average of 6.77 IV's per patient, or 1.16 IV's per patient per month.

Parameters monitored in all patients were Total Forced Vital Capacity (FVC), Forced Expiratory Flow in 1 second (FVC 1.0), Peak Expiratory Flow (PEF), Forced Expiratory Flow through $25 \%$ to $75 \%$ of exhalation (FEF $25-75 \%$ ) and Forced Expiratory Flow through $75 \%$ to $85 \%$ of exhalation (FEF 75-85\%).

Preservative-free nutrients were used without exception, and there were no serious systemic and no local reactions at any time during therapy. Early in the study some patients experienced lightheadedness, nausea or near fainting with rapid IV infusions. This was secondary to magnesium-induced hypotension, and this problem was recognized early in the study and avoided.

\section{Results}

Results were measured as percentage improvement of the percentage predicted on the Knudsen scale. For example, if the result of a parameter of the pre-infusion pulmonary function test was calculated as $50 \%$ of predicted normal, and IV treatment resulted in a value of $75 \%$ of predicted normal for that same parameter, the percentage improve- ment for that particular parameter would be entered as $50 \%$.

The 38 pre-infusion/post-infusion patients demonstrated an average improvement of $28 \%$ in FVC, $44 \%$ in FEV 1 , $44 \%$ in PEF, $54 \%$ in FEF $25-75$ and $38 \%$ in FEF $75-85$, with an overall average improvement of $45 \%$ (Tables 2,3 ).

The 13 patients measured for improvement over time (sequential data, average duration 5.82 months with an average of 1.66 infusions/patient/mo.) for chronic asthma showed an average improvement of $50 \%$ in $\mathrm{FVC}$, $57 \%$ in FEV $1,64 \%$ in PEF, 64\% in FEF $25-75$ and $44 \%$ in FEF 75-85, with an overall average improvement of $57 \%$ (Tables 4,5).

Of the 13 patient multiple infusion group, 4 patients who received Short Term therapy (infusions for an average duration of a month or less) for chronic asthma demonstrated an average improvement of $40 \%$ in $\mathrm{FVC}, 43 \%$ in FEV $1,45 \%$ in PEF, 36\% in FEF $25-75$ and $6 \%$ in FEF $75-$ 85 , with an overall average improvement of $34 \%$ (Table 6). Nine (9) patients who received Long Term therapy (infusions for an average duration of 12.58 months) for chronic asthma demonstrated an average improvement of $59 \%$ in FVC, $87 \%$ in FEV $1,88 \%$ in PEF, $128 \%$ in FEF $25-$ 75 and $113 \%$ in FEF $75-85$, with an overall average improvement of 95\% (Table 7).

\section{Discussion}

Although there were no age-matched control patients in this outcome study, and certainly uncontrolled variables, considerable improvement was observed after parenteral infusion therapy with multiple nutrients, both for pre/ post treatment and treatment over time. There was more pronounced improvement after longer-term treatment. Patients who received treatment for longer than a month 
Table 2: Pre-/Post IV Treatment Data - 39 Patients Percent Predicted, Pre- and Post- infusion with \% improvement

\begin{tabular}{|c|c|c|c|c|c|c|c|c|c|c|c|c|c|c|c|c|c|}
\hline \multirow[b]{3}{*}{ Patient } & \multirow{3}{*}{ Sex } & \multirow{3}{*}{ Age } & \multicolumn{3}{|c|}{ FVC (L) } & \multicolumn{3}{|c|}{ FEV I (L) } & \multicolumn{3}{|c|}{ PEF (L/S) } & \multicolumn{3}{|c|}{ FEF 25-75 (L/S) } & \multicolumn{3}{|c|}{ FEF 75-85 (L/S) } \\
\hline & & & \multicolumn{3}{|c|}{$\%$ Predicted } & \multicolumn{3}{|c|}{$\%$ Predicted } & \multicolumn{3}{|c|}{$\%$ Predicted } & \multicolumn{3}{|c|}{$\%$ Predicted } & \multicolumn{3}{|c|}{$\%$ Predicted } \\
\hline & & & Pre & Post & $\%$ & Pre & Post & $\%$ & Pre & Post & $\%$ & Pre & Post & $\%$ & Pre & Post & $\%$ \\
\hline$A, L$. & $M$ & 55 & 36 & 72 & $100 \%$ & 36 & 79 & $119 \%$ & 64 & 92 & $44 \%$ & 35 & 129 & $269 \%$ & 32 & 112 & $250 \%$ \\
\hline$A, S$. & $\mathrm{F}$ & 48 & 28 & 50 & $79 \%$ & 30 & 48 & $60 \%$ & 40 & 78 & $95 \%$ & 30 & 40 & $33 \%$ & 40 & 38 & $-5 \%$ \\
\hline$B, M$. & $\mathrm{F}$ & 57 & 69 & 100 & $45 \%$ & 48 & 81 & $69 \%$ & 45 & 115 & $156 \%$ & 24 & 39 & $63 \%$ & 30 & 32 & $7 \%$ \\
\hline$B, R$. & $M$ & 46 & 73 & 93 & $27 \%$ & 69 & 87 & $26 \%$ & 95 & 106 & $12 \%$ & 57 & 70 & $23 \%$ & 66 & 67 & $2 \%$ \\
\hline$B, V$. & $\mathrm{F}$ & 39 & 34 & 67 & $97 \%$ & 34 & 72 & III2\% & 51 & 59 & $16 \%$ & 38 & 96 & $153 \%$ & 26 & 98 & $277 \%$ \\
\hline$B, V$ & $\mathrm{~F}$ & 70 & 38 & 54 & $42 \%$ & 32 & 47 & $47 \%$ & 32 & 53 & $66 \%$ & 18 & 30 & $67 \%$ & 25 & 34 & $36 \%$ \\
\hline C, D. & $M$ & 59 & 27 & 30 & II\% & 14 & 17 & $21 \%$ & 10 & 12 & $20 \%$ & 10 & 10 & $0 \%$ & 32 & 28 & $-13 \%$ \\
\hline C, A. & $\mathrm{F}$ & 33 & 90 & 91 & $1 \%$ & 80 & 98 & $23 \%$ & 115 & 142 & $23 \%$ & 60 & 108 & $80 \%$ & 65 & 116 & $78 \%$ \\
\hline C, K. & $\mathrm{F}$ & 35 & 45 & 62 & $38 \%$ & 34 & 52 & $53 \%$ & 32 & 56 & $75 \%$ & 22 & 36 & $64 \%$ & 32 & 41 & $28 \%$ \\
\hline $\mathrm{D}, \mathrm{C}$. & $F$ & 33 & 65 & 65 & $0 \%$ & 49 & 56 & $14 \%$ & 62 & 65 & $5 \%$ & 31 & 41 & $32 \%$ & 42 & 51 & $21 \%$ \\
\hline $\mathrm{D}, \mathrm{B}$. & $M$ & 49 & 65 & 80 & $23 \%$ & 72 & 83 & $15 \%$ & 68 & 88 & $29 \%$ & 75 & 80 & $7 \%$ & 146 & 147 & $1 \%$ \\
\hline $\mathrm{D}, \mathrm{K}$. & $\mathrm{F}$ & 48 & 61 & 74 & $21 \%$ & 56 & 70 & $25 \%$ & 89 & 95 & $7 \%$ & 39 & 60 & $54 \%$ & 29 & 33 & $14 \%$ \\
\hline $\mathrm{F}, \mathrm{L}$. & $M$ & 58 & 26 & 29 & $12 \%$ & 12 & 13 & $8 \%$ & 7 & 8 & $14 \%$ & 7.4 & 8.1 & $9 \%$ & 22 & 26 & $18 \%$ \\
\hline G, W. & $M$ & 74 & 48 & 67 & $40 \%$ & 25 & 57 & $128 \%$ & 16 & 88 & $450 \%$ & 11 & 37 & $236 \%$ & 38 & 63 & $66 \%$ \\
\hline G, B. & $M$ & 45 & 83 & 113 & $36 \%$ & 83 & 101 & $22 \%$ & 94 & 149 & $\mathbf{5 9} \%$ & 81 & 70 & $-14 \%$ & 89 & 60 & $-33 \%$ \\
\hline G, G. & $\mathrm{F}$ & 42 & 83 & 93 & $12 \%$ & 46 & 82 & $78 \%$ & 24 & 59 & $146 \%$ & 31 & 66 & $113 \%$ & 55 & 79 & $44 \%$ \\
\hline G, L. & $\mathrm{F}$ & 63 & 50 & 78 & $56 \%$ & 36 & 60 & $67 \%$ & 52 & 71 & $37 \%$ & 17 & 29 & $71 \%$ & 21 & 29 & $38 \%$ \\
\hline J, E. & $\mathrm{F}$ & 63 & 47 & 85 & $81 \%$ & 38 & 84 & $121 \%$ & 43 & 64 & $49 \%$ & 19 & 79 & $316 \%$ & 20 & 55 & $175 \%$ \\
\hline $\mathrm{K}, \mathrm{H}$. & $\mathrm{F}$ & 49 & 58 & 72 & $24 \%$ & 59 & 72 & $22 \%$ & 82 & 99 & $21 \%$ & 53 & 62 & $17 \%$ & 76 & 86 & $13 \%$ \\
\hline M, A. & $F$ & 46 & 85 & 97 & $14 \%$ & 79 & 89 & $13 \%$ & 61 & 86 & $41 \%$ & 71 & 71 & $0 \%$ & 73 & 68 & $-7 \%$ \\
\hline$M, W$ & $M$ & 85 & 74 & 77 & $4 \%$ & 69 & 90 & $30 \%$ & 46 & 82 & $78 \%$ & 50 & 119 & $138 \%$ & 148 & 458 & $209 \%$ \\
\hline M, C. & $\mathrm{F}$ & 36 & 93 & 85 & $-9 \%$ & 52 & 53 & $2 \%$ & 30 & 46 & $53 \%$ & 26 & 25 & $-4 \%$ & 26 & 25.5 & $-2 \%$ \\
\hline M, J. & $M$ & 64 & 74 & 75 & $1 \%$ & 49 & 57 & $16 \%$ & 47 & 60 & $28 \%$ & 25 & 37 & $48 \%$ & 57 & 83 & $46 \%$ \\
\hline $\mathrm{O}, \mathrm{M}$ & $\mathrm{F}$ & 49 & 90 & 99 & $10 \%$ & 98 & 114 & $16 \%$ & 88 & 167 & $90 \%$ & 114 & 168 & $47 \%$ & 145 & 214 & $48 \%$ \\
\hline P, O. & $\mathrm{F}$ & 47 & 54 & 72 & $33 \%$ & 52 & 76 & $46 \%$ & 85 & 84 & $-1 \%$ & 46 & 82 & $78 \%$ & 40 & 74 & $85 \%$ \\
\hline $\mathrm{R}, \mathrm{B}$. & $F$ & 69 & 21 & 23 & $10 \%$ & 16 & 19 & $19 \%$ & 13 & 17 & $31 \%$ & 10 & 11 & $10 \%$ & 21 & 22 & $5 \%$ \\
\hline S, M. & $\mathrm{F}$ & 60 & 56 & 94 & $68 \%$ & 45 & 88 & $96 \%$ & 60 & 104 & $73 \%$ & 26 & 66 & $154 \%$ & 26 & 51 & $96 \%$ \\
\hline S, J. & $M$ & 54 & 98 & 166 & $69 \%$ & 76 & 96 & $26 \%$ & 77 & 70 & $-9 \%$ & 34 & 39 & $15 \%$ & 70 & 90 & $29 \%$ \\
\hline $\mathrm{S}, \mathrm{S}$ & $M$ & 40 & 73 & 85 & $16 \%$ & 58 & 69 & $19 \%$ & 68 & 85 & $25 \%$ & 36 & 40 & $11 \%$ & 43 & 38 & $-12 \%$ \\
\hline S, D. & $\mathrm{F}$ & 64 & 40 & 56 & $40 \%$ & 25 & 33 & $32 \%$ & 18 & 21 & $17 \%$ & 15 & 14 & $-7 \%$ & 42 & 34 & $-19 \%$ \\
\hline S, W. & $M$ & 44 & 71 & 80 & $13 \%$ & 52 & 65 & $25 \%$ & 42 & 60 & $43 \%$ & 31 & 40 & $29 \%$ & 31 & 38 & $23 \%$ \\
\hline $\mathrm{T}, \mathrm{P}$ & $\mathrm{F}$ & 45 & 41 & 59 & $44 \%$ & 26 & 46 & $77 \%$ & 22 & 73 & $232 \%$ & 17 & 27 & $\mathbf{5 9} \%$ & 26 & 32 & $23 \%$ \\
\hline$W, P$. & $\mathrm{F}$ & 65 & 48 & 76 & $\mathbf{5 8} \%$ & 17 & 31 & $82 \%$ & 9 & 21 & $133 \%$ & 9 & 11 & $22 \%$ & 20 & 19 & $-3 \%$ \\
\hline W, B. & $M$ & 38 & 54 & 72 & $33 \%$ & 42 & 58 & $38 \%$ & 32 & 46 & $44 \%$ & 25 & 37 & $48 \%$ & 37 & 50 & $35 \%$ \\
\hline$W, R$. & $M$ & 40 & 77 & 77 & $0 \%$ & 34 & 41 & $21 \%$ & 27 & 33 & $22 \%$ & 12 & 16 & $33 \%$ & 21 & 24 & $14 \%$ \\
\hline W, C. & $\mathrm{F}$ & 55 & 28 & 39 & $39 \%$ & 20 & 28 & $40 \%$ & 17 & 26 & $53 \%$ & 12 & 16 & $33 \%$ & 20 & 23 & $15 \%$ \\
\hline W, G. & $\mathrm{F}$ & 70 & 101 & 108 & $7 \%$ & 78 & 98 & $26 \%$ & 34 & 82 & $141 \%$ & 51 & 65 & $27 \%$ & 49 & 62 & $27 \%$ \\
\hline Z, A. & $\mathrm{F}$ & 43 & 62 & 75 & $21 \%$ & 64 & 75 & $17 \%$ & 107 & 87 & $-19 \%$ & 68 & 88 & $29 \%$ & 57 & 49 & $-14 \%$ \\
\hline Average: & & 52 & 60 & 76 & $28 \%$ & 48 & 65 & $38 \%$ & 50 & 72 & $44 \%$ & 35 & 54 & $54 \%$ & 48 & 70 & $44 \%$ \\
\hline \multicolumn{5}{|c|}{ Average Overall Improvement } & $45 \%$ & & & & & & & & & & & & \\
\hline
\end{tabular}

Results were measured as percentage improvement of the percentage predicted on the Knudsen scale

Table 3: Summary, Pre- and Post-IV Treatment Spirometry Results - 39 patients (Acute Asthma Protocol) Acute Asthma Pre- and Post-infusion Values Measured in Percent Predicted

\begin{tabular}{llll}
\hline Parameter & Pre-IV & Post-IV & Ave. \% Improved \\
\hline FVC (L) & 60 & 76 & $28 \%$ \\
FEV I (L) & 48 & 65 & $38 \%$ \\
PEF (L/S) & 50 & 72 & $44 \%$ \\
FEF 25-75 (L/S) & 35 & 54 & $54 \%$ \\
FEF 75-85 (L/S) & 48 & 70 & $44 \%$ \\
Total Average Improvement & & & $\mathbf{4 5 \%}$ \\
\hline
\end{tabular}

Results were measured as percentage improvement in the percentage predicted on the Knudsen scale 
Table 4: Trend Data - I3 Patients

\begin{tabular}{|c|c|c|c|c|c|c|c|c|c|c|c|c|c|c|c|c|c|c|c|}
\hline \multirow{3}{*}{ Patient } & \multirow[b]{3}{*}{ Sex } & \multirow[b]{3}{*}{ Age } & \multirow[b]{3}{*}{$\begin{array}{l}\text { Mos } \\
\text {. }\end{array}$} & \multirow[b]{3}{*}{$\begin{array}{l}\text { \# IVs } \\
\text { per pt. }\end{array}$} & \multicolumn{3}{|c|}{$\%$ Predicted } & \multicolumn{3}{|c|}{$\%$ Predicted } & \multicolumn{3}{|c|}{$\%$ Predicted } & \multicolumn{3}{|c|}{$\%$ Predicted } & \multicolumn{3}{|c|}{$\%$ Predicted } \\
\hline & & & & & \multicolumn{3}{|c|}{ FVC (L) } & \multicolumn{3}{|c|}{ FEV I (L) } & \multicolumn{3}{|c|}{ PEF (L/S) } & \multicolumn{3}{|c|}{ FEF $25-75$ (L/S) } & \multicolumn{3}{|c|}{ FEF 75-85 (L/S) } \\
\hline & & & & & Pre & Post & $\%$ & Pre & Post & $\%$ & Pre & Post & $\%$ & Pre & Post & $\%$ & Pre & Post & $\%$ \\
\hline$B, M$. & $\mathrm{F}$ & 57 & 9.5 & 11 & 73 & 97 & $33 \%$ & 56 & 78 & $39 \%$ & 89 & 109 & $22 \%$ & 26 & 41 & $58 \%$ & 29 & 40 & $38 \%$ \\
\hline$B, C$ & $M$ & 31 & 2.3 & 8 & 34 & 97 & $185 \%$ & 39 & 69 & $77 \%$ & 30 & 53 & $\mathbf{7 7} \%$ & 17 & 45 & $165 \%$ & 13 & 52 & $300 \%$ \\
\hline $\mathrm{D}, \mathrm{K}$ & $\mathrm{F}$ & 16 & 5.3 & 11 & 119 & 143 & $20 \%$ & 94 & 96 & $2 \%$ & 95 & 134 & $41 \%$ & 54 & 37 & $-31 \%$ & \multicolumn{3}{|c|}{ Not done } \\
\hline G, W. & $M$ & 74 & 3.5 & 4 & 48 & 67 & $40 \%$ & 25 & 57 & $128 \%$ & 16 & 93 & $481 \%$ & 11 & 34 & $209 \%$ & 38 & 54 & $42 \%$ \\
\hline G, L. & $\mathrm{F}$ & 63 & 3 & 4 & 50 & 82 & $64 \%$ & 36 & 65 & $81 \%$ & 51 & 56 & $10 \%$ & 17 & 35 & $106 \%$ & 21 & 36 & $71 \%$ \\
\hline $\mathrm{H}, \mathrm{K}$. & $\mathrm{F}$ & 55 & 5.3 & 11 & 61 & 105 & $72 \%$ & 41 & 97 & $137 \%$ & 56 & 120 & $1 \mid 4 \%$ & 18 & 38 & $111 \%$ & 20 & 43 & $115 \%$ \\
\hline L, L. & $\mathrm{F}$ & 53 & 0.3 & 2 & 40 & 87 & $118 \%$ & 42 & 69 & $64 \%$ & 50 & 47 & $-6 \%$ & 38 & 47 & $24 \%$ & 62 & 49 & $-21 \%$ \\
\hline O, M. & $\mathrm{F}$ & 48 & 19 & 4 & 72 & 90 & $25 \%$ & 63 & 98 & $56 \%$ & 60 & 88 & $47 \%$ & 42 & 114 & $171 \%$ & 42 & 145 & $245 \%$ \\
\hline $\mathrm{P}, \mathrm{R}$. & $M$ & 77 & I & 6 & 65 & 88 & $35 \%$ & 66 & 89 & $35 \%$ & 73 & 92 & $26 \%$ & 57 & 80 & $40 \%$ & 165 & $|7|$ & $4 \%$ \\
\hline $\mathrm{R}, \mathrm{B}$. & $\mathrm{F}$ & 49 & 0.2 & 3 & 59 & 72 & $22 \%$ & 59 & 71 & $20 \%$ & 44 & 80 & $82 \%$ & 61 & 70 & $15 \%$ & 67 & 49 & $-27 \%$ \\
\hline S, L. & $\mathrm{F}$ & 64 & 10 & 9 & 61 & 86 & $41 \%$ & $4 I$ & 64 & $56 \%$ & 73 & 113 & $\mathbf{5 5} \%$ & 16 & 24 & $\mathbf{5 0} \%$ & 27 & 22 & $-19 \%$ \\
\hline S, W. & $M$ & 44 & 16 & 13 & 45 & 98 & $118 \%$ & 35 & 77 & $120 \%$ & 34 & 98 & $188 \%$ & 21 & 45 & $114 \%$ & 31 & 46 & $48 \%$ \\
\hline W, G. & $\mathrm{F}$ & 70 & 0.2 & 2 & 65 & 74 & $14 \%$ & 38 & 64 & $68 \%$ & 16 & 47 & $194 \%$ & 20 & 42 & $110 \%$ & 21 & 64 & $205 \%$ \\
\hline Average & & 54 & 5.82 & 6.77 & 61 & 91 & $\mathbf{5 0} \%$ & 49 & 76 & $\mathbf{5 7} \%$ & 53 & 87 & $64 \%$ & 31 & 50 & $64 \%$ & 45 & 64 & $44 \%$ \\
\hline \multicolumn{5}{|c|}{ Ave. \# IVs per patient per month } & 1.16 & & & & & & & & & & & & & & \\
\hline \multicolumn{5}{|c|}{ Average Overall Improvement } & $57 \%$ & & & & & & & & & & & & & & \\
\hline
\end{tabular}

Results were measured as percentage improvement in the percentage predicted on the Knudsen scale

Table 5: Trends in Patients with Chronic Asthma - 13 patients Trend Values Measured in Percent Predicted

\begin{tabular}{llll}
\hline Parameter & Beginning of Therapy & Conclusion of Therapy & Ave. \% Improved \\
\hline FVC (L) & 61 & 91 & $50 \%$ \\
FEV I (L) & 49 & 76 & $57 \%$ \\
PEF (L/S) & 53 & 87 & $64 \%$ \\
FEF 25-75 (L/S) & 31 & 50 & $64 \%$ \\
FEF 75-85 (L/S) & 45 & 64 & $44 \%$ \\
Total Average Improvement & & & $\mathbf{5 7 \%}$
\end{tabular}

Trend values were obtained by subtracting the results of the first spirometry testing in the series from the results of the last (most current) in the series. Results were measured as percentage improvement in the percentage predicted on the Knudsen scale.

fared considerably better than those who stopped therapy within a month or less.

Patients experienced rapid clinical relief during - or at some time after - most infusions, depending on their degree of distress, and as the data demonstrates, pulmonary function improved overall during the sequential infusion study. It would also appear that the effectiveness of this type of therapy might be cumulative, as patients appeared to require treatment less often as time passed. Drug usage was decreased in all patients, and discontinued or reduced to intermittent use in over half of the patients studied.

The primary reason mixed nutrients were employed in this study is that prior to this study the author had observed that the effect of a combination of nutrients was consistently more beneficial than the infusion of IV magnesium sulfate alone. Patients given magnesium (sulfate) alone also seemed to develop a more rapidly increasing tolerance for (or resistance to) magnesium, and pulmonary function did not improve nearly as significantly as were those of patients who were given the complete protocols. Therefore, infusions of magnesium sulfate alone, without other nutrients added, was not employed in this study.

It has been demonstrated previously that intravenous infusions with magnesium sulfate appear to be more effective that nebulized albuterol alone [26], and it has also been demonstrated that intravenous magnesium may be successful when all other more "traditional" interventions, including corticosteroids, have failed [28]. Consid- 
Table 6: Short-term Trend Data (infusions for one month or less) 4 Patients

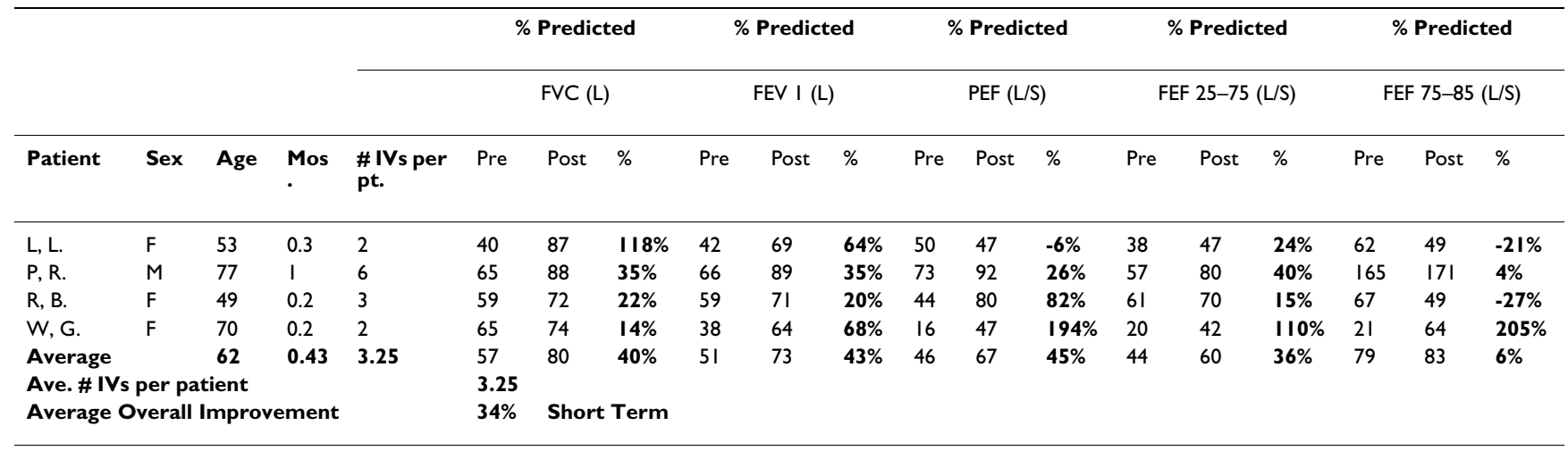

Results were measured as percentage improvement in the percentage predicted on the Knudsen scale

Table 7: Long-term Trend Data (infusion for over a month) 9 Patients

\begin{tabular}{|c|c|c|c|c|c|c|c|c|c|c|c|c|c|c|c|c|c|c|c|}
\hline \multirow{3}{*}{ Patient } & \multirow[b]{3}{*}{ Sex } & \multirow[b]{3}{*}{ Age } & \multirow[b]{3}{*}{ Mos. } & \multirow[b]{3}{*}{$\begin{array}{l}\text { \# IVs } \\
\text { Per pt. }\end{array}$} & \multicolumn{3}{|c|}{$\%$ Predicted } & \multicolumn{3}{|c|}{$\%$ Predicted } & \multicolumn{3}{|c|}{$\%$ Predicted } & \multicolumn{3}{|c|}{$\%$ Predicted } & \multicolumn{3}{|c|}{$\%$ Predicted } \\
\hline & & & & & \multicolumn{3}{|c|}{ FVC (L) } & \multicolumn{3}{|c|}{ FEV I (L) } & \multicolumn{3}{|c|}{ PEF (L/S) } & \multicolumn{3}{|c|}{ FEF 25-75 (L/S) } & \multicolumn{3}{|c|}{ FEF 75-85 (L/S) } \\
\hline & & & & & Pre & Post & $\%$ & Pre & Post & $\%$ & Pre & Post & $\%$ & Pre & Post & $\%$ & Pre & Post & $\%$ \\
\hline$B, M$. & $\mathrm{F}$ & 57 & 9.5 & 11 & 73 & 97 & $33 \%$ & 56 & 78 & $39 \%$ & 89 & 109 & $22 \%$ & 26 & 41 & $58 \%$ & 29 & 40 & $38 \%$ \\
\hline$B, C$ & $M$ & 31 & 2.3 & 8 & 34 & 97 & $185 \%$ & 39 & 69 & $77 \%$ & 30 & 53 & $77 \%$ & 17 & 45 & $165 \%$ & 13 & 52 & $300 \%$ \\
\hline $\mathrm{D}, \mathrm{K}$ & $\mathrm{F}$ & 16 & 5.3 & 11 & 119 & 143 & $20 \%$ & 94 & 96 & $2 \%$ & 95 & 134 & $41 \%$ & 54 & 37 & $-31 \%$ & $\begin{array}{l}\text { Not } \\
\text { done }\end{array}$ & & \\
\hline G, W. & $M$ & 74 & 3.5 & 4 & 48 & 67 & $40 \%$ & 25 & 57 & $128 \%$ & 16 & 93 & $481 \%$ & 11 & 34 & $209 \%$ & 38 & 54 & $42 \%$ \\
\hline G, L. & $\mathrm{F}$ & 63 & 3 & 4 & 50 & 82 & $64 \%$ & 36 & 65 & $81 \%$ & 51 & 56 & $10 \%$ & 17 & 35 & $106 \%$ & 21 & 36 & $71 \%$ \\
\hline $\mathrm{H}, \mathrm{K}$. & $\mathrm{F}$ & 55 & 5.3 & II & 61 & 105 & $72 \%$ & $4 I$ & 97 & $137 \%$ & 56 & 120 & $114 \%$ & 18 & 38 & III\% & 20 & 43 & $115 \%$ \\
\hline O, M. & $\mathrm{F}$ & 48 & 19 & 4 & 72 & 90 & $25 \%$ & 63 & 98 & $56 \%$ & 60 & 88 & $47 \%$ & 42 & 114 & $171 \%$ & 42 & 145 & $245 \%$ \\
\hline S, L. & $\mathrm{F}$ & 64 & 10 & 9 & 61 & 86 & $41 \%$ & 41 & 64 & $56 \%$ & 73 & 113 & $55 \%$ & 16 & 24 & $50 \%$ & 27 & 22 & $-19 \%$ \\
\hline S, W. & $M$ & 44 & 16 & 13 & 45 & 98 & $118 \%$ & 35 & 77 & $120 \%$ & 34 & 98 & $188 \%$ & 21 & 45 & $114 \%$ & 31 & 46 & $48 \%$ \\
\hline Average & & 53 & 12.58 & 9.25 & 60 & 95 & $59 \%$ & 45 & 84 & $87 \%$ & 56 & 105 & $88 \%$ & 24 & 55 & $128 \%$ & 30 & 64 & $113 \%$ \\
\hline \multicolumn{5}{|c|}{ Ave. \# IVs per patient per month } & $\begin{array}{l}0.7 \\
4\end{array}$ & & & & & & & & & & & & & & \\
\hline \multicolumn{5}{|c|}{ Average Overall Improvement } & $\begin{array}{l}95 \\
\%\end{array}$ & \multicolumn{2}{|c|}{ Long Term } & & & & & & & & & & & & \\
\hline
\end{tabular}

Results were measured as percentage improvement in the percentage predicted on the Knudsen scale

ering this, the results of this study came as no surprise to the author.

There are numerous metabolic and biochemical explanations as to why each of the specific nutrients added might indeed provide more benefit for asthmatics than magnesium sulfate alone. For example, Vitamin $\mathrm{C}$ is known to have a general antihistaminic effect [39], it decreases bronchial responsiveness overall [40] and bronchial responsiveness to histamine in patients with allergic rhinitis [41]. Vitamin C is also a potent free radical scavenger, and free radicals are known to play a role in the cause of airway obstruction attendant with asthma [42].
Trace minerals also mitigate the inflammatory response [43], perhaps because they play a major role in the antioxidation of free radicals [44]. Further, manganese has been found deficient in bronchial biopsies of asthmatic patients, indication manganese replenishment could aid in the treatment of asthma. [45].

Lastly, zinc is an essential trace mineral for most immune mechanisms in the body to function, including lymphocyte (T-cell) function [46].

This reasoning could partly explain why the patients in this study who received longer-term therapy with infusions fared best. Molybdenum, as Wright's study demonstrated [36], may be a significant part of the long-term 
benefit. Indeed, those patients who received an average of less than one infusion monthly for over a year fared almost three times as well as those who received infusions for a month or less.

Over the period of this study, the author observed that 6 of 44 asthmatic patients appeared to fail with this particular type of infusion therapy. These 6 patients, however, were judged as failures prior to any patient having received three IV treatments. Since this study was undertaken, the author has found that it sometimes takes 3 to 5 infusions for patients to observe a substantial clinical benefit. Therefore, actual failure may be in question.

Shortly after this study was first begun, it became clear that patients who received weekly or more frequent therapy with these parenteral nutrients for a period of $11 / 2$ to 3 months improved more rapidly and distinctly than patients who received only occasional infusions. It also became evident that this group of patients was able to extend the interval between treatments to 8-14 days and then longer. Several patients were able to extend their intervals out as far as 4-6 months with little apparent loss of efficacy.

This led the author to reach the preliminary conclusion that there may be some type of "loading" period, or dose accumulation, of one or more of these nutrients employed here. Dr. Wright, working with intravenous molybdenum for asthma, discovered this same phenomenon [36].

It was also often observed that patients with acute asthma sometimes did not improve immediately, or even appeared to worsen immediately after an infusion. This is very likely secondary to the acute bronchodilitation resulting from this treatment, with resultant mucous production (release) and coughing. Studies have shown that optimal pulmonary function is likely to occur considerably later than 10 minutes after an infusion of magnesium, even out to perhaps 80-110 minutes [10]. The author's experience supports this observation and it is likely that the post-infusion measurements in this study were usually taken before the maximum benefit occurred.

As a more important note, the author observed that the Infusion protocol, when given as the initial treatment, whether for a patient with acute or chronic asthma, could cause considerable worsening of symptoms several hours after the infusion. Early in the study, three patients who were given the Infusion as their first treatment experienced increased respiratory distress later in the day, two of which required emergency treatment. The author postulates that the infusion administration of either the molybdenum or the trace minerals (containing molybdenum) prior to "priming" a patient with at least one IV that does not contain minerals can cause an adverse reaction. Although the mechanism of this reaction is not yet clear, the author speculates this response may be secondary to an acute sulfite detoxification response to molybdenum.

Considering this, the author advises that neither trace minerals nor molybdenum be included in any rapidly infused IV or in any IV to be administered for acute asthma.

The author would conclude that parenteral infusion therapy with the nutrients used in this study might have considerable benefit. Infusions of these particular parental nutrients very often made the difference for patients who otherwise were responding poorly to other modalities of treatment.

As a result of this study, the author is of the opinion that this type of therapy adds a dimension to the treatment of chronic asthma not attainable with conventional therapy using bronchodilators and corticosteroids, especially in the acute situation.

In a time when multiple factors appear to be coalescing to increase both the incidence and morbidity of asthma around the world, it would seem that any therapy that adds a measure of exclusion from these statistics should be considered whenever possible.

\section{Acknowledgements}

Susan Ung, RN, Peggy Maltese, Jana Boggs, Allee Jay, RN, Diane Menzies, Patti Robinson and Barbara Shrader for their help in collecting the data for this study.

\section{References}

I. Sly RM, O'Donnell R: Stabilization of asthma mortality. Comment in Ann Allergy Asthma Immunol 1997, 78(4):347-54. Ann. Aller. Asthma \& Immunol. 1997;78 (4): 347-54.

2. Lang DM: Trends in US asthma mortality: good news and bad news. Comment on: Ann Allergy Asthma Immunol 1997, 78(4):333-7. Ann. Aller. Asthma Immunol. 1997;78(4): 333-7.

3. Lieberman JS, Kane GC: Asthma mortality: the worldwide response. J R Soc Med 1997, 90(5):265-7.

4. Beasley R, Nishima S, Pearce N, Crane J: Beta-agonist therapy and asthma mortality in Japan [letter]. Lancet 1998, 35 I (9 | | 3): | 406-7.

5. Keistinen T, Saynajakangas O, Tuuponen T, Kivela S, Ostro B, Chestnut $L$ : Assessing the health benefits of reducing particulate matter air pollution in the United States. Environ Research 1998, 76(2):94-106.

6. Ertle A, London $\mathrm{M}$ : Insights into asthma prevalence in Oregon. J Asthma 1998, 35(3):28I-9.

7. Mannino DM, Homa DM, Pertowski CA, Ashizawa A, Nixon LL, Johnson CA, Ball LB, Jack E, Kang DS: Surveillance for asthma United States 1960-1995. MMWR CDC Surveill Summ 1998, 47(I): I-27.

8. Nakazawsa T, Kawakami Y, Sudo M, Kobayashi S, Suetsugu S, Nakajima S, Yamakido $M$, Nagano $H$ : [Trends of asthma death among adults in Japan 1992-1994. Analysis of 313 cases reported questionnaires sent to hospitals with more than 100 beds]. Arerugi - Jap J Allergology 1998, 47(I):4I-7. 
9. Okayama H, Aikawa T, Okayama M, Sasaki H, Mue S, Takishima T: Bronchodilating effect of intravenous magnesium sulfate in bronchial asthma. JAMA 1987, 257(8): 1076-78.

10. Rolla G, Bucca C, Caria E, Arossa W, Bugiani M, Cesano L, Caropreso $A$ : Acute effect of intravenous magnesium sulfate on airway obstruction of asthmatic patients. Ann Allerg 1988, 6I(5):388-9I.

II. McNamara R, Spivey W, Skobeloff E, Jacubowitz S: Intravenous magnesium sulfate in the management of acute respiratory failure complicating asthma. Ann Emerg Med 1989, I8(2): 197-9.

12. Bone R, Burch S: Management of status asthmaticus. Ann Allerg I99|, 67(5):46|-9.

13. Noppen M, Vanmaele L, Impens N, Schandevyl W: Bronchodilating effect of intravenous magnesium sulfate in acute severe bronchial asthma. Chest 1990, 97(2):373-6.

14. Kuitert L, Kletchko S: Intravenous magnesium sulfate in acute, life-threatening asthma. Ann Emerg Med I991, 20(II):1243-5. [published erratum appears in Ann. Emerg. Med. 1992 Oct, 2I(I0): 1272].

15. Green SM, Rothrock SG: Intravenous magnesium for acute asthma: failure to decrease emergency treatment duration or need for hospitalization [see comments]. Ann Emerg Med 1992:260-5.

16. Tiffany B, Berk W, Todd I, White S: Magnesium bolus or infusion fails to improve expiratory flow in acute asthma exacerbations. Chest 1993, 104(3):83I-4.

17. Rolla G, Bucca C, Brussino L, Colagrande P: Effect of intravenous magnesium infusion on salbutamol-induced bronchodilatation in patients with asthma. Magnesium Research 1994, 7(2):129-33.

18. Lemesle FG: High-dose intravenous magnesium sulfate in the management of life-threatening status asthmaticus. Intensive Care Med 1995, 2 I(1):94-5.

19. Sydow M, Crozier T, Zielmann S, Radke J, Burchardi H: High-dose intravenous magnesium sulfate in the management of lifethreatening status asthmaticus. Intensive Care Med 1993, | 9(8):467-7|.

20. Schiermeyer R, Finkelstein J: Rapid infusion of magnesium sulfate obviates need for intubation in status asthmaticus. Amer J Emer Med 1994, I2(2): 164-6.

21. Bloch H, Silverman R, Mancherje N, Grant S, Jagminas L, Scharf S: Intravenous magnesium sulfate as an adjunct in the treatment of acute asthma [see comments]. Chest 1995, 07(6): I576-8. Chest 1995;07(6): $1576-8 \mid$

22. Keen J: Intravenous magnesium sulfate for acute asthma. J Emerg Nurs 1995, 2 I( I):44-6.

23. Hill J, Britton J: Effect of intravenous magnesium sulpfate on airway calibre and airway reactivity to histamine in asthmatic subjects. Br J Clin Pharmacol 1996, 42(5):629-3I.

24. Downey $P$, Cox $R$ : Update on the management of status asthmaticus. Curr Opin Pediatr 1996, 8(3):226-33.

25. Ciarallo L, Sauer A, Shannon M: Intravenous magnesium therapy for moderate to severe pediatric asthma: results of a randomized, placebo-controlled trial [see comments]. J Ped 1996, I 29(6):809-14. J. Ped. 1996; I29(6): 809-14.

26. Devi PR, Kumar L, Singhi S, Prasad R, Singh M: Intravenous magnesiumsulfate in acute severe asthma not responding to conventional therapy. Indian Pediatr 1997, 34(5):389-97.

27. Frakes MA, Richardson LE: 2 nd. Magnesium sulfate therapy in certain emergency conditions. Am J of Emerg Med 1997, I5(2): 182-7.

28. Mills $R$, Leadbeater $M$, Ravalia $A$ : Intravenous magnesium sulphate in the management of refractory bronchospasm in a ventilated asthmatic. Anaesthesia 1997, 52(8):782-5.

29. Gurkan F, Haspolat K, Bosnak M, Dikici B, Derman O, Ece A: Intravenous magnesium sulphate in the management of moderate to severe acute asthmatic children non-responding to conventional therapy. Eur J Emerg Med 1999, 6(3):20I-5.

30. Swain R, Kaplan-Machlis B: Magnesium for the next millennium. South Med J 1999, 92(I I): 1040-7.

31. Alter H, Koepsell T, Hilty WM: Intravenous magnesium as an adjuvant in acute bronchospasm: a meta-analysis. Ann Emerg Med 2000, 36(3):191-7. Comment in: Ann Emerg Med. 2000;36(3):234-6.
32. Ciarallo L, Brousseau D, Reinert S: Higher-dose intravenous magnesium therapy for children with moderate to severe acute asthma. Arch Pediatr Adolesc Med 2000, I 54( I 0):979-83.

33. Rowe BH, Bretzlaff JA, Bourdon C, Bota GW, Camargo CA Jr: Magnesium sulfate for treating exacerbations of acute asthma in the emergency department. Cochrane Database Syst Rev 2000:CD00I490.

34. Schenk P, Vonbank K, Schnack B, Haber P, Lehr S, Smetana R: Intravenous magnesium sulfate for bronchial hyperreactivity: a randomized, controlled, double-blind study. Clin Pharmacol Ther 200I, 69(5):365-7I.

35. Silverman RA, Osborn H, Runge J, Gallagher El, Chiang W, Feldman J, Gaeta T, Freeman K, Levin B, Mancherje N, Scharf S: IV magnesium sulfate in the treatment of acute severe asthma: a multicenter randomized controlled trial. Chest 2002, I 22(2):489-97. Erratum in: Chest 2002;122(5):1870. Comment in: Chest. 2002; 122(2):396-8.

36. Wright J., Littleton K: Defects in sulfur metabolism. Internatl Clin Nut $\operatorname{Rev} 1989,9(3): 118-19$.

37. Wright J: Vitamin B-I2: Powerful protection against childhood asthma. Internat/ Clin Nutr Rev 1989, 9(4):185-8.

38. Anibarro $B$ : Asthma with sulfite intolerance in children: a blocking study with cyanocobalamin. J Allerg Clin Immunol 1992, 90:103-9.

39. Johnston C: Antihistamine effect of supplemental ascorbic acid in neutrophil chemotaxis. J Am Coll Nutr 1992, II(2): 172-6.

40. Bucca C: Effect of Vitamin C on transient increase of bronchial responsiveness in conditions affecting the upper respiratory airways. Beyond Deficiency: New Views on the Function and Health Effects of Vitamins, New York Academy of Sciences. 1992:9-12.

41. Bucca C: Effect of vitamin C on histamine bronchial responsiveness of patients with allergic rhinitis. Ann Allergy 1990, 65:311-14.

42. Kanazawa $\mathrm{H}$ : The role of free radicals in airway obstruction in asthmatic patients. Chest 1991, 100:1319-22.

43. McClain C: Minerals and inflammatory response. J Am Coll Nutr 1992, I I(5):598 Abstract 4.

44. Zidenberg-Cherr S: Essential trace elements in antioxidant processes. Trace Elements, Micronutrients and Free Radicals 1992:107-27.

45. Campbell M: Low levels of manganese in bronchial biopsies from asthmatic subjects. J Aller Clin Immunol 1992, 89(I, Part II):332-749.

46. Prasad A: Zinc and lymphocyte immune function. J Am Coll Nutr 1992, I I(5):567 Abstract 3.
Publish with Bio Med Central and every scientist can read your work free of charge

"BioMed Central will be the most significant development for disseminating the results of biomedical research in our lifetime. "

Sir Paul Nurse, Cancer Research UK

Your research papers will be:

- available free of charge to the entire biomedical community

- peer reviewed and published immediately upon acceptance

- cited in PubMed and archived on PubMed Central

- yours - you keep the copyright
BioMedcentral 